\title{
The Disturbance of Melanogenesis and Melanosome Transfer in the Leukoderma Lesions of Extramammary Paget's Disease
}

\author{
Atsushi Tanemura ${ }^{1}$, Aya Tanaka', Fei Yang ${ }^{1}$, Eiji Kiyohara ${ }^{1}$, Yorihisa Kotobuki ${ }^{1}$, \\ Mari Wataya-Kaneda', Naoki Oiso² ${ }^{2}$, Ichiro Katayama1 \\ ${ }^{1}$ Department of Dermatology, Graduate School of Medicine, Osaka University, Osaka, Japan \\ ${ }^{2}$ Department of Dermatology, Faculty of Medicine, Kinki University, Osaka, Japan \\ Email: tanemura@derma.med.osaka-u.ac.jp
}

How to cite this paper: Tanemura, A., Tanaka, A., Yang, F., Kiyohara, E., Kotobuki, Y., Wataya-Kaneda, M., Oiso, N. and Katayama, I. (2018) The Disturbance of Melanogenesis and Melanosome Transfer in the Leukoderma Lesions of Extramammary Paget's Disease. Journal of Cosmetics, Dermatological Sciences and Applications, 8, 10-13.

https://doi.org/10.4236/jcdsa.2018.81003

Received: January 1, 2018

Accepted: March 6, 2018

Published: March 9, 2018

Copyright ( 92018 by authors and Scientific Research Publishing Inc. This work is licensed under the Creative Commons Attribution International License (CC BY 4.0).

http://creativecommons.org/licenses/by/4.0/

(c) (i) Open Access

\begin{abstract}
We frequently encounter characteristic color variation including hypopigmentation, hyperpigmentation, and erythema in extramammary Paget's disease (EMPD) lesions. Owing to unclear hypopigmentation, the lesional border of EMPD can be poorly defined and it is likely insufficient to perform its complete resection. Although the existence of Toker's cells and lack of lesional bFGF production have been reported to cause hypopigmentation inside of EMPD lesions, exact mechanisms of hypopigmentation in EMPD are not fully explored. We experienced three EMPD patients with obviously hypopigmented EMPD macules and histopathologically confirmed a reduced number of melanocytes on the hypopigmented macules and their loss on the erythematous plaques or nodules. An ultrastructural analysis on the hypopigmented lesions revealed disturbance of melanosome maturation and melanosome transfer to the adherent Pagets' cell on the basal layer. No Paget's cells even adhered to remaining melanocytes with dendrites contained matured melanosome and a few number of matured melanosome complexes were observed in basal keratinocytes. In the present study, we hypothesize that severe disturbance of not only melanogenesis but also melanosome transfer to surrounding Paget's cells and basal keratinocytes may cause characteristic hypopigmentation in EMPD. Future bioanalysis would reveal molecular mechanisms for hypopigmentation in EMPD.
\end{abstract}

\section{Keywords}

Hypopigmentation, Extramammary Paget's Disease (EMPD), Melanocyte to Paget's Cell Interaction, Ultrastructural Analysis, Mechanism 


\section{Introduction}

Extramammary Paget's disease (EMPD) is a cutaneous malignancy arising on the genital and/or perianal regions that presents with various features including scaly erythema, hyperpigmentation, and hypopigmentation. In particular, due to hypopigmented macule on the periphery of EMPD lesions that show centrifugal enlargement, it can be difficult to recognize the lesion border (Figure 1(a)). Although several mechanisms have been proposed to underlie the characteristic hypopigmentation, none of these hypothesized mechanisms has been fully accepted [1] [2] [3]. Dermoscopic observation has shown that-in comparison to other skin malignancies-a milky-red area is observed significantly more frequently in EMPDs [4]. In the present study, we evaluated the microscopic and ultrascopic features of hypopigmented lesions in detail.

(a)

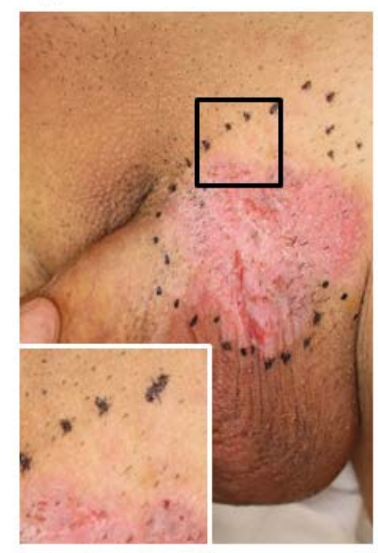

(c)
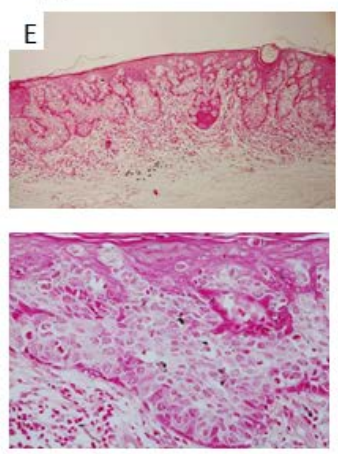

(b)

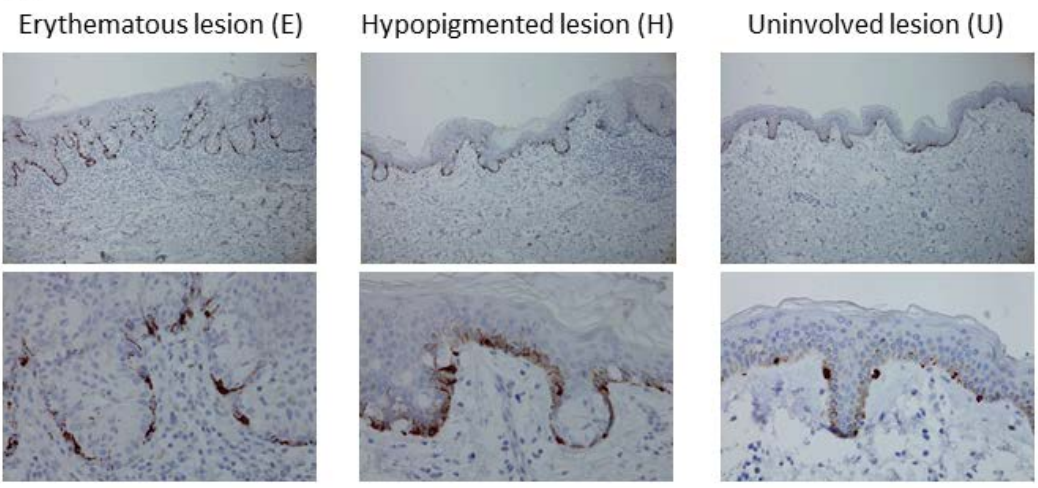

(d)

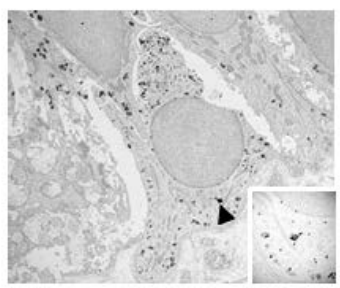

(e)

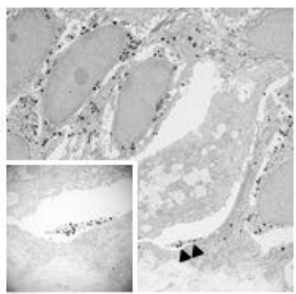

Figure 1. (a) The clinical features of a hypopigmented lesion on the periphery of EMPD (the box indicates an enlarged view); (b) Melan-A-positive melanocytes remained in both the hypopigmented and erythematous EMPD lesions. The magnification levels of the upper and lower images are $\times 40$ and $\times 200$, respectively; (c) The gradual decrease in the basal melanosomes from hypopigmented to erythematous lesions. The magnification levels of the upper and lower images are $\times 40$ and $\times 200$, respectively; (d) The ultrastructural features of the melanocytes in the EMPD lesion. Fewer matured melanosomes were detected in the remaining melanocytes (magnification, $\times 15,000$; arrowhead). The box shows a matured melanosome surrounded by black colloid particles (magnification, $\times 60,000$ ); (e) The ultrastructural features of melanocytes and the adjacent Paget's cells in the EMPD lesion. No melanosome transfer to the Paget's cell was observed (magnification, $\times 15,000$; arrowheads). The box indicates a melanocyte's dendrite containing a matured melanosome (magnification, $\times 60,000$ ). 


\section{Materials and Methods}

Specimens of hypopigmented and erythematous lesions in EMPD from 3 patients were simultaneously processed for histopathological, immunohistochemical, and ultrastructural analyses. Melanocytes were detected by Melan A and HMB 45 staining and melanosome was visualized by Masson-Fontana staining.

\section{Results and Discussion}

Although the number of Melan-A-positive melanocytes was decreased, Melan-A-positive melanocytes were still present on the hypopigmented lesions. In contrast, far fewer Melan-A-positive melanocytes were observed on the erythematous plaques (Figure 1(b)). Fontana-Masson staining showed basal melanosis skipping the bottom of Paget's cell clusters, which might have been associated with the white-colored appearance of EMPD lesion. In contrast, no deposition of melanosomes was found on the erythematous plaques (Figure 1(c)). The infiltration of $\mathrm{CD}^{+}$and $\mathrm{CD}^{+}$inflammatory $\mathrm{T}$ cells into the erythematous plaques was far greater than that in the hypopigmented macules and perilesions; however, incontinentia pigmenti and apoptotic melanocytes were not evident. Next, melanocytes (from the sites that were used for the histopathological analysis) were stained for Pmel-17/TRP-1 and examined under an electron microscope. Electron microscopy revealed a notable decrease in the number of matured melanosomes among the remaining melanocytes and that the melanocyte dendrites were elongated, likely to allow the transfer of matured melanosomes to the neighboring keratinocytes and Paget's cells (Figure 1(d)). In comparison to the perilesions, globules containing matured melanosomes were less evident in the basal keratinocytes on hypopigmented macules. Interestingly, melanosomes were delivered to the basal keratinocytes, while prevented to the Paget's cells beyond the melanocytes (Figure 1(e)). This is thought to represent direct evidence of severe disturbance in melanosome transfer to Paget's cells. The number of remaining melanocytes in the erythematous lesions was much lower in comparison to the peripheral hypopigmented lesions. This finding indicates that the remaining melanocytes may gradually disappear in accordance with a progression of disease and that hypopigmentation could be an initial sign of the onset of EMPD. Skipped clusters of Paget's cells did not receive any melanosomes from the neighboring melanocytes in the basal layer.

To our knowledge, this is the $1^{\text {st }}$ report to analyze the ultrastructure of the melanocytes and the formation of melanosomes in EMPD lesions. We hypothesize that severe disturbance of not only melanogenesis but also melanosome transfer to the surrounding Paget's cells and basal keratinocytes may cause the characteristic hypopigmentation in EMPD. Juxtacrine factors from Paget's cells may interfere in melanogenesis and in the survival of melanocytes. A future in vitro bioanalysis should help to reveal the precise molecular mechanisms responsible for hypopigmentation in EMPD. 


\section{References}

[1] Chen, Y.H., Wong, T.W. and Lee, Y.Y. (2001) Depigmented Genital Extrammary Paget's Disease: A Possible Histogenetic Link to Toker's Clear Cells and Clear Cell Papulosis. Journal of Cutaneous Pathology, 28, 105-108. https://doi.org/10.1034/j.1600-0560.2001.280208.x

[2] Yang, C.C., Lee, J.Y. and Wong, T.W. (2004) Depigmented Extramammary Paget's Disease. British Journal of Dermatology, 151, 1049-1053. https://doi.org/10.1111/j.1365-2133.2004.06223.x

[3] Chiba, H., Takazawa, T., Takenouchi, T., Nomoto, S., Yamada, S., Tago, O. and Ito, M. (2000) Two Cases of Vulval Pigmented Extrammary Paget's Disease: Histochemical and Immunohistochemical Studies. British Journal of Dermatology, 142, 1190-1194. https://doi.org/10.1046/j.1365-2133.2000.03547.x

[4] Mun, J.H., Park, S.M., Kim, G.W., Song, M., Kim, H.S., Ko, H.C., Kim, B.S. and Kim, M.B. (2016) Clinical and Dermoscopic Characteristics of Extrammary Paget Disease: A Study of 35 Cases. British Journal of Dermatology, 174, 1104-1107. https://doi.org/10.1111/bjd.14300 